\title{
On the measurement of socioeconomic inequality of health between countries
}

\author{
Guido Erreygers ${ }^{1,2}$ (D) Philip Clarke ${ }^{2}$ Q Qiong Zheng ${ }^{2}$
}

Received: 4 May 2016 / Accepted: 28 November 2016 / Published online: 21 January 2017

(C) The Author(s) 2017. This article is published with open access at Springerlink.com

\begin{abstract}
This paper focuses on the measurement of socioeconomic inequality of health between countries and its evolution over time, by means of population-weighted indicators. We show that rank-dependent indicators of inequality can be highly sensitive to small changes in the socioeconomic variable when estimating inequality in samples consisting of countries with large differences in population weights. Since larger countries count more than smaller countries, changes in the former tend to have bigger effects than changes in the latter. When using rank-dependent indicators, however, the sensitivity to small changes in the variable which is used for ranking can be so extreme, that the indicator may suggest trend reversals in inequality which do not really exist. An empirical study shows that this is not a freak case. The use of rank-dependent indicators may therefore produce misleading results when it comes to the measurement of population-weighted between-country inequality. We propose a simple diagnostic test to check how sensitive rank-dependent indices are to small changes in the variable used for ranking.
\end{abstract}

Keywords Inequality measurement · Socioeconomic inequality of health · Concentration index

Guido Erreygers

guido.erreygers@uantwerpen.be

Philip Clarke

philip.clarke@unimelb.edu.au

Qiong Zheng

qiong.zheng@unimelb.edu.au

1 Department of Economics, University of Antwerp, Antwerp, Belgium

2 Centre for Health Policy, University of Melbourne, Melbourne, Australia 


\section{Introduction}

High-income countries are not only richer, but in general also healthier than low-income countries. The famous Preston curve traces a positive, concave relationship between GDP per capita and life expectancy (Preston, 1975), and forcefully illustrates the fact that people in richer countries tend to live longer than people in poorer countries. Over time, most countries have moved upwards along the Preston curve, and moreover the curve itself has shifted upwards (Deaton, 2013, chapter 1). Both evolutions mean that on average people live longer and enjoy higher incomes than they did in the past. While the shape and location of the curve certainly provide useful information on income-related inequality in health around the world, it does not come with an associated aggregate measure of inequality that can be used to assess whether health is becoming more or less unequally distributed between poorer and richer countries. Given the increasing interest in the measurement of global inequality and the improving availability of data, we expect to see more and more studies focusing on the measurement of global income-related inequality of health. It seems safe to assume that these studies will apply the methods that are nowadays widely used to measure income-related health inequality across individuals within a country.

Up to now, just a few isolated attempts have been made to apply these methods to the measurement of income-related inequality of health across countries or regions. By far the most popular indicator when it comes to the measurement of income-related health inequality is the Concentration index, a rank-dependent indicator which is closely related to the Gini coefficient. It has been used for the measurement of income-related inequality of health both between regions of the world (Reidpath and Allotey, 2007) and between countries of a region (Hajizadeh et al., 2014). While the former study considers disabilityadjusted life years (DALYs) as its health variable and reports a single value for the Concentration index, the latter focuses on the prevalence of HIV/ AIDS and calculates both relative and generalised Concentration indices (for the population as a whole, for men and women, and for the rural and urban population) to gain more insight into socioeconomic inequality of HIV/AIDS in sub-Saharan Africa. In related work, Dorling, Shaw and Smith (2006) quantify changes in life expectancy between high and low income regions of the world since 1950, using the slope index of inequality. The slope index of inequality is closely related to the generalised Concentration index (Wagstaff, Paci and Van Doorslaer, 1991: 550), but often preferred by epidemiologists as they deem it easy to interpret.

The contribution of this paper is to show that a problem arises when using a rankdependent index to measure income-related health inequality between countries of vastly different size. As argued by Sala-i-Martin (2006: 354), the measurement of between-country inequality necessitates the use of population weights: larger countries, such as China and India, should count more than smaller countries, such as Luxemburg or Monaco. In the literature on global income inequality this is known as "Concept 2" inequality (Milanovic, 2013: 199). Although in some studies of global inequality a case is made for unweighted, or "Concept 1" inequality (see, e.g., Decancq, Decoster and Schokkaert, 2009), we deal exclusively with population-weighted inequality. This means that we use the weighted version of the Concentration index (O'Donnell et al., 2008: Chapter 8), which resembles the weighted version of the Gini coefficient (Lerman and Yitzhaki, 1989; Creedy, 2015). In the weighted versions, the (fractional) income ranks are replaced by the weighted (fractional) income ranks.

The main point of our paper is that if there are large differences in population weights which is almost inevitable when using country data on a global scale - the Concentration index becomes highly sensitive to small changes in the variable which is used for ranking. 
Small changes in per capita incomes can lead to large swings in the measured degree of income-related inequality. An empirical application shows that this is more than a theoretical curiosity, and may produce misleading conclusions when it comes to assessing trends in inequality.

The sensitivity to which we draw attention here is only partly due to the rank-dependent character of the index. We illustrate this by comparing how a small change affects the Gini coefficient and how it affects the Concentration index. What we learn from this comparison is that the sensitivity problem identified here can be seen as the outcome of an explosive combination of rank-dependency and population weighting in the measurement of incomerelated inequality of health.

\section{The effect of a small change in income on the Gini coefficient}

Assume we are considering a sample of $n$ countries, denoted by $1,2, \ldots, n$. For each country $i$ we know the income level $y_{i}$ (e.g., GDP per capita), which we assume to be nonnegative. We denote the income distribution by $y=\left(y_{1}, y_{2}, \ldots, y_{n}\right)$, and for convenience we label countries in ascending order of income, i.e. $y_{1} \leq y_{2} \leq \ldots \leq y_{n}$. The sample weight of country $i$, here measured by the share of country $i$ in the total population of the sample, is equal to $s_{i}$.

To begin with, let us assume that all countries have the same sample weight, i.e. $1 / n$. If no two countries have the same income level, the income rank $r_{i}$ of country $i$ is simply $i$. If there are ties in the income distribution, the income rank is equal to the average of the tied group. In other words, if we have $y_{j-1}<y_{j}=\ldots=y_{j+k-1}<y_{j+k}$, each of the $k$ countries $j, \ldots, j+k-1$ has income rank $j+(k-1) / 2$.

In the case of equal sample weights, the absolute version of the Gini coefficient can be written as:

$$
G^{*}=\frac{1}{n} \sum_{i=1}^{n} \frac{2 r_{i}-n-1}{n} y_{i} .
$$

An equivalent expression can be found by using the fractional rank $q_{i}$, which is defined as:

$$
q_{i}=\left(r_{i}-\frac{1}{2}\right) / n
$$

It is easy to see that Eq. 1 can be rewritten as:

$$
G^{*}=\frac{1}{n} \sum_{i=1}^{n}\left(2 q_{i}-1\right) y_{i} .
$$

The relative version of the Gini coefficient is equal to the absolute version divided by the average income level $\mu_{y}$, i.e. $G=G^{*} / \mu_{y}$.

When not all countries have the same sample weights, we need to redefine the fractional ranks and the index. If country $i$ is not tied with any other, its fractional rank is equal to:

$$
q_{i}=\frac{s_{i}}{2}+\sum_{l=0}^{i-1} s_{l}
$$


where we take $s_{0}=0$. If country $i$ belongs to a group of tied countries $j, \ldots, j+k-1$, its fractional rank is equal to:

$$
q_{i}=\frac{\sum_{l=j}^{j+k-1} s_{l}}{2}+\sum_{l=0}^{j-1} s_{l} .
$$

Given either Eq. 4 or Eq. 5, the formula of the index now becomes:

$$
G^{*}=\sum_{i=1}^{n} s_{i}\left(2 q_{i}-1\right) y_{i} .
$$

These expressions allow us to derive how a change in income affects the index. Let us concentrate here on the simplest case which can be imagined, that in which the income of only one country increases, from $y_{i}$ to $y_{i}^{\prime}=y_{i}+\delta$, with $\delta>0$ (see Hoffmann, 2001, and Lambert and Lanza, 2006, for a broader and more general analysis of this change). We distinguish three cases, according to whether the change in income is smaller than, equal to, or higher than the distance to country $i+1$ 's income.

Let $y_{i+1}-y_{i}=d>0$. To begin with, we assume that $\delta<d$, i.e. $y_{i}+\delta<y_{i+1}$. There are no changes in ranks, but the index changes as a result of the change in income of country $i$. In fact, as long as the ranks remain the same, the change in the index $\Delta G^{*}=G^{* \prime}-G^{*}$ is equal to:

$$
\Delta G^{*}=s_{i}\left(2 q_{i}-1\right) y_{i}^{\prime}-s_{i}\left(2 q_{i}-1\right) y_{i} .
$$

This can be simplified to:

$$
\Delta G^{*}=s_{i}\left(2 q_{i}-1\right) \delta .
$$

As $\delta$ increases but remains lower than $d$, the index changes in a smooth way: it decreases if country $i$ is located in the lower half of the income distribution (i.e., where $q_{i}<0.5$ ), and increases if $i$ is located in the upper half $\left(q_{i}>0.5\right)$. Moreover, the effect will be proportional to the size of the country, its location in the distribution, and the magnitude of the change.

At some point, however, the change will be so large that a change in ranks will occur. The second case we have to examine is the one in which $\delta=d$, i.e. $y_{i}+\delta=y_{i+1}$. In that case, countries $i$ and $i+1$ are tied, and applying Eq. 5 we find that:

$$
q_{i}^{\prime}=q_{i}+\frac{s_{i+1}}{2}=q_{i+1}-\frac{s_{i}}{2}=q_{i+1}^{\prime} .
$$

The effect on the index is now a result of the change in the ranks of countries $i$ and $i+1$ and of the change in the level of income of country $i$. To be precise, the effect is equal to:

$$
\Delta G^{*}=s_{i}\left(2 q_{i}^{\prime}-1\right) y_{i}^{\prime}-s_{i}\left(2 q_{i}-1\right) y_{i}+s_{i+1}\left(2 q_{i+1}^{\prime}-1\right) y_{i+1}-s_{i+1}\left(2 q_{i+1}-1\right) y_{i+1} \text {. }
$$

Working out the terms, and taking into account that in this case $y_{i+1}-y_{i}=\delta=d$, we can simplify Eq. 10 to:

$$
\Delta G^{*}=s_{i}\left(2 q_{i}-1\right) d .
$$

Given that Eq. 11 is equal to Eq. 8 when $\delta=d$, this means that at the moment in which the ranks change, the index does not jump brutally: its change is in line with what it was before the change in ranks.

But what if $\delta>d$, i.e. $y_{i}+\delta>y_{i+1}$ ? Applying Eq. 4, we derive that the relations between the fractional ranks before and after the change are now:

$$
\begin{gathered}
q_{i}^{\prime}=q_{i}+s_{i+1} \\
q_{i+1}^{\prime}=q_{i+1}-s_{i} .
\end{gathered}
$$


Fig. 1 The effect of $\delta$ on $G^{*}\left(q_{i}>1 / 2\right)$

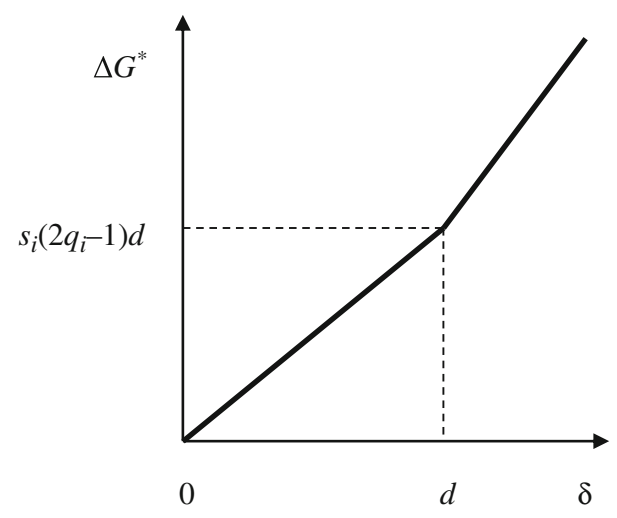

The change in the value of the index is again equal to Eq. 10. Working out the terms, we now obtain:

$$
\Delta G^{*}=s_{i}\left(2 q_{i}-1\right) \delta+2 s_{i} s_{i+1}(\delta-d) .
$$

In comparison to Eqs. 8 and 11, there is an additional term which comes from the change in the ranks of countries $i$ and $i+1$. As $\delta$ increases, the index changes smoothly, but slightly differently than before. (For $0<\delta<d$ we have $\Delta G^{*} / \Delta \delta=s_{i}\left(2 q_{i}-1\right.$ ), and for $d<\delta$ we have $\Delta G^{*} / \Delta \delta=s_{i}\left(2 q_{i}-1\right)+2 s_{i} s_{i+1}$.) Whether the effect on the index is now positive or negative, depends not only on the fractional rank of country $i$, but also on the size of country $i+1$ and on the magnitudes of the differences between the incomes of $i$ and $i+1$ before and after the change. If country $i$ is initially located in the upper half of the income distribution $\left(q_{i} \geq 0.5\right)$, it is certain that the effect is positive. If, however, country $i$ is initially located in the bottom half of the distribution, the effect may be both positive and negative. In the middle of the distribution and just below it, as long as $q_{i}>\frac{1}{2}-\frac{s_{i+1}(\delta-d)}{\delta}$, the effect is positive; in the opposite case, it is negative. The size of the effect depends on the weights of the two countries affected by the change, on the location of country $i$ in the income distribution, and on the magnitudes of the differences between the incomes of the two countries before and after the change.

To sum up, as long as small changes in the income of one country do not disturb the ranks, they affect the index in a smooth way, according to a linear function, with the sign of the effect determined by the position of the country in the income distribution and the size by the weight of the country and by its position in the income distribution. As soon as there is a rank switch between two countries, the effect on the index is still described by a linear function, but one with a different shape than before. A graphical illustration can be found in Fig. 1, where we assume that country $i$ belongs to the top half of the distribution (i.e., $q_{i}>1 / 2$ ). At the moment in which the switch occurs, the index does not make a jump; the switch coincides with the point of intersection between two linear curves and constitutes a kink point. ${ }^{1}$

The effect on the relative version of the index, i.e. the Gini coefficient $G=G^{*} / \mu_{y}$, is equal to:

$$
\Delta G=\frac{\mu_{y} \Delta G^{*}-\Delta \mu_{y} G^{*}}{\mu_{y}\left(\mu_{y}+\Delta \mu_{y}\right)}
$$

\footnotetext{
${ }^{1}$ In formal terms, we could say that the Gini coefficient $G^{*}(y)$ is continuous in income, in the sense that $\lim _{y \rightarrow y^{\circ}} G^{*}(y)=G^{*}\left(y^{\circ}\right)$ for any nonnegative $y^{\circ}$.
} 
where $\Delta G^{*}$ is as defined before and $\Delta \mu_{y}=s_{i} \delta$. If $\Delta G^{*} \leq 0$, we certainly have $\Delta G<0$; if $\Delta G^{*}>0$, however, we have $\Delta G<0$ when $\Delta G^{*}<\left(\Delta \mu_{y} / \mu_{y}\right) G^{*}=\Delta \mu_{y} G$ and $\Delta G>0$ in the opposite case. ${ }^{2}$

\section{The effect of a small change in income or health on the Concentration index}

We now shift our attention to the measurement of socioeconomic inequality. Suppose that for each country $i$ we know the income level $y_{i}$ and the health level $h_{i}$, both of which we assume to be nonnegative, ratio-scale variables. ${ }^{3}$ The income and health distributions are now $y=\left(y_{1}, y_{2}, \ldots, y_{n}\right)$ and $h=\left(h_{1}, h_{2}, \ldots, h_{n}\right)$. We continue to label countries in ascending order of income, i.e. $y_{1} \leq y_{2} \leq \ldots \leq y_{n}$.

The Concentration index is based on the ranks of countries in the income distribution and their levels in the health distribution. When all countries have the same sample weight, equal to $1 / n$, the absolute version of the Concentration index can be written as:

$$
C^{*}=\frac{1}{n} \sum_{i=1}^{n} \frac{2 r_{i}-n-1}{n} h_{i} .
$$

Using the fractional rank $q_{i}$, we can rewrite Eq. 16 as:

$$
C^{*}=\frac{1}{n} \sum_{i=1}^{n}\left(2 q_{i}-1\right) h_{i} .
$$

In the presence of sample weights, the formula of the index becomes:

$$
C^{*}=\sum_{i=1}^{n} s_{i}\left(2 q_{i}-1\right) h_{i} .
$$

The relative version of the index is equal to the absolute version divided by the average health level $\mu_{h}$, i.e. $C=C^{*} / \mu_{h}$.

Since the Concentration index looks at the correlation between two variables, two types of changes can be examined: a change in income, and a change in health. The effect of a change in health is the easiest to explore. Suppose that the health level of country $i$ changes from $h_{i}$ to $h_{i}+\varepsilon$, with $\varepsilon>0$, while everything else remains unchanged. The effect on the absolute Concentration index is similar to that of a non-rank-affecting income change on the Gini coefficient. We therefore have:

$$
\Delta C^{*}=s_{i}\left(2 q_{i}-1\right) \varepsilon .
$$

As far as the relative value of the Concentration index is concerned, we likewise have:

$$
\Delta C=\frac{\mu_{h} \Delta C^{*}-\Delta \mu_{h} C^{*}}{\mu_{h}\left(\mu_{h}+\Delta \mu_{h}\right)} .
$$

\footnotetext{
${ }^{2}$ For a non-rank-affecting change in income, this means that $\Delta G>0$ if and only if $q_{i}>(1+G) / 2$; this result has been shown by Hoffmann (2001: 243) and Lambert and Lanza (2006: 261). For a rank-affecting change, the condition becomes $q_{i}>(1+G) / 2-s_{i+1}(\delta-d) / \delta$.

${ }^{3}$ It seems useful to point out that health variables are often not of the ratio-scale type. Inequality measurement is, however, not limited to ratio-scale variables. For instance, for bounded cardinal variables modified versions of the usual inequality indicators have been suggested (see, e.g., Erreygers and Van Ourti, 2011).
} 
Fig. 2 The effect of $\delta$ on $C^{*}\left(h_{i}>h_{i+1}\right)$

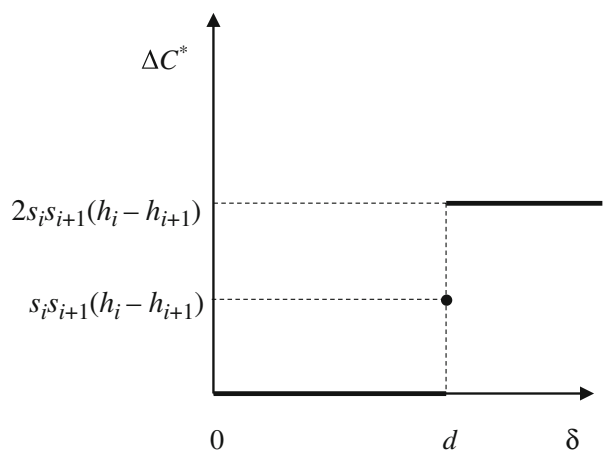

Let us now switch to the analysis of an income change. As before, we assume that the income of a single country changes from $y_{i}$ to $y_{i}+\delta$, and we distinguish three cases: $0<\delta<d, \delta=d$ and $d<\delta$, where $d=y_{i+1}-y_{i}$. The first case is extremely simple: the index remains unchanged. Since all weights $s_{i}$, fractional ranks $q_{i}$ and health levels $h_{i}$ remain the same, it can be seen from Eq. 18 that the index is not affected. Hence, we have:

$$
\Delta C^{*}=0 .
$$

If $\delta=d$, however, things do change. As we have seen before, countries $i$ and $i+1$ are now tied, and their new ranks are given by Eq. 9. The change in the index is equal to:

$$
\Delta C^{*}=s_{i}\left(2 q_{i}^{\prime}-1\right) h_{i}-s_{i}\left(2 q_{i}-1\right) h_{i}+s_{i+1}\left(2 q_{i+1}^{\prime}-1\right) h_{i+1}-s_{i+1}\left(2 q_{i+1}-1\right) h_{i+1} .
$$

Working out the terms, we can simplify this to:

$$
\Delta C^{*}=s_{i} s_{i+1}\left(h_{i}-h_{i+1}\right) .
$$

This means that the index makes a sudden jump, with the sign of the change determined by the sign of the difference between the health levels of the two countries, and the size by the weights of the two countries and by the difference between their health levels.

The final case is the one where $d<\delta$. The changes in the fractional ranks are given by Eqs. 12 and 13. The effect on the index is given by Eq. 22, which can be reduced to:

$$
\Delta C^{*}=2 s_{i} s_{i+1}\left(h_{i}-h_{i+1}\right) .
$$

Comparing Eq. 24 to Eq. 23, we see that a second jump occurs, of exactly the same size as the previous one. The effect on the index is independent of the magnitude of the change in income; it depends on the product of the sample weights $s_{i}$ and $s_{i+1}$, and on the difference between the health levels $h_{i}$ and $h_{i+1}$. Figure 2 represents what happens in the three cases, under the assumption that $h_{i}>h_{i+1} \cdot{ }^{4}$

The effect on the relative version of the Concentration index is simply:

$$
\Delta C=\frac{\Delta C^{*}}{\mu_{h}} .
$$

The reaction of the Concentration index to a change in income is remarkably different from the reaction of the Gini coefficient. Table 1 summarizes how the two absolute indices change

\footnotetext{
${ }^{4}$ More formally, the Concentration index is continuous in health, but not in income, i.e. we have $\lim _{h \rightarrow h^{\circ}} C^{*}\left(y^{\circ}, h\right)=C^{*}\left(y^{\circ}, h^{\circ}\right)$ for any nonnegative $y^{\circ}$ and $h^{\circ}$, but there exist nonnegative $y^{\circ}$ for which $\lim _{y \rightarrow y^{\circ}} C^{*}\left(y, h^{\circ}\right) \neq C^{*}\left(y^{\circ}, h^{\circ}\right)$.
} 
Table 1 The reactions of $G^{*}$ and $C^{*}$ to a change in income of individual $i$

\begin{tabular}{lll}
\hline & $\Delta G^{*}$ & $\Delta C^{*}$ \\
\hline $0<\delta<d$ & $s_{i}\left(2 q_{i}-1\right) \delta$ & 0 \\
$\delta=d$ & $s_{i}\left(2 q_{i}-1\right) d$ & $s_{i} s_{i+1}\left(h_{i}-h_{i+1}\right)$ \\
$d<\delta$ & $s_{i}\left(2 q_{i}-1\right) \delta+s_{i} s_{i+1}(\delta-d)$ & $2 s_{i} s_{i+1}\left(h_{i}-h_{i+1}\right)$ \\
\hline
\end{tabular}

in the three cases we have examined. The Gini coefficient reacts to the smallest change of income, even in the absence of any changes in ranks; the Concentration index, by contrast, only reacts when there are changes in ranks. While the sign of the change of the Gini coefficient depends exclusively (before changes in ranks) or predominantly (after changes in ranks) upon the position of $i$ in the income distribution, that of the Concentration index depends exclusively upon the difference between the health levels of the countries with changing ranks. Finally, whereas the Gini coefficient reacts smoothly (albeit with a kink at the moment in which the ranks change), the Concentration index reacts brutally: it jumps when the ranks change.

In reality one will seldom find changes which affect only the ranks of two successive countries in the income distribution. If the other changes are relatively minor, however, it may very well be that the effect of a small change in income levels dominates the effects of other changes on the index. This is what we show below.

\section{Income and life expectancy}

There is solid empirical evidence showing that richer people tend to be in better health and live longer than those who are poor. What we are interested in here, is to what extent this relationship holds across nations. It is, of course, widely known that the average citizen of a high-income country usually lives longer than the average citizen of a poor country, and often much longer. What we would like to find out is whether there is a long-term trend in this cross-country socioeconomic inequality of life expectancy.

As mentioned before, the well-known Preston curve graphically illustrates the positive association between countries' GDP per capita and life expectancy (Preston, 1975). The available evidence strongly suggests the existence of a non-linear relationship between income and life expectancy: for poorer countries the slope of the Preston curve appears to be much steeper than it is for richer countries. The dividing line or "hinge point" (Deaton, 2013: 31) is said to correspond to the epidemiological transition, which separates countries where infectious diseases, especially among children, are the main causes of death, from countries where chronic diseases, especially among older people, are the main causes of death.

Indices measuring the socioeconomic inequality of life expectancy may be thought of as indicators of the average or aggregate degree of correlation between income and life expectancy. Put differently, these indices reflect the overall slope of the curve, by expressing what may be called the 'global social gradient' in a single number. If the relationship is of a non-linear kind, however, it would perhaps be better to have two numbers instead of just one: one index for low-income countries, where the gradient appears to be fairly steep, and another for high-income countries, where it appears to be much flatter. In what follows we therefore report index values for the world as a whole, and for the subsets of low- and highincome countries. To split our sample into these two subsets, we use the weighted average 
Table 2 Summary data on the whole sample

\begin{tabular}{lllll}
\hline & $\begin{array}{l}1960 \\
\text { Sample }\end{array}$ & World & $\begin{array}{l}2011 \\
\text { Sample }\end{array}$ & World \\
\hline Population (persons) & $2,514,136,320$ & $3,034,970,564$ & $5,935,228,800$ & $7,007,432,419$ \\
GDP/capita (2005 US \$)* & 3,567 & - & 11,073 & - \\
Life expectancy at birth (years) & 51.3 & 52.5 & 71.1 & 70.5 \\
\hline
\end{tabular}

Source: World Bank, World Development Indicators and own calculations based on Penn World Table 8.1

* Calculated based on Penn World Table expenditure-side real GDP at chained PPPs

of GDP per capita for the world as a whole. Drawing the boundary there is arbitrary, of course, but at least it gives us a rough indication of the gradient among poorer and richer countries. The main question we are interested in is whether the global social gradient has become steeper or flatter over time. In addition, we would like to know whether anything has changed within and between the groups of low- and high-income countries.

Since our sample consists of countries, we use population size weights equal to the shares of the countries in the overall population of the sample. Our income variable is GDP per capita, where GDP is measured as expenditure-side real GDP at chained PPPs (in 2005 US\$). Our health variable is life expectancy at birth. Population and life expectancy data come from the World Development Indicators of the World Bank, while GDP data are obtained from Penn World Table 8.1. We arrived at a sample of 103 countries for which all data were available on a yearly basis for the period 1960-2011. As can be seen from Table 2, our sample seems fairly representative for the world as a whole. It contains more than 80 $\%$ of the world population, and both the 1960 and 2011 average life expectancy are close to the world averages as reported by the World Bank.

\section{The global social gradient}

To measure the evolution of the socioeconomic inequality of life expectancy across countries for the world as a whole, we use the absolute version of the Concentration index, $C^{*}$. 5 Figure 3 represents the evolution of the index between 1960 and 2011. From 1960 to $1976 C^{*}$ decreased firmly and almost constantly (minor increases occurred only in 1966, 1973 and 1975). In 1977, however, there is sudden bump in the graph, indicating a dramatic increase in inequality. The value of $C^{*}$ jumped from 2.1407 to 3.5595 , which is roughly the level of inequality reached in 1967 . From 1977 to 1988 the index tended to increase, and after that it decreased very slowly. In 2011 the value of the index was almost the same as it was in 1977. Although the index decreased between the beginning and the end of the period, no uniform trend can be detected.

Let us zoom in on the rather brutal bump of 1977, which is of a magnitude suggesting an earthquake-like trend reversal. Nevertheless, if one takes a closer look at the data, nothing much seems to have happened in 1977 , and certainly nothing indicating a spectacular change in the course of events. How can we explain this puzzle?

The mechanism behind the leap in the value of $C^{*}$ lies in the relative positions of China and India, and to a lesser extent Indonesia. In 1976 the per capita GDP of China was slightly

\footnotetext{
5 The use of the relative version of the index changes very little.
} 


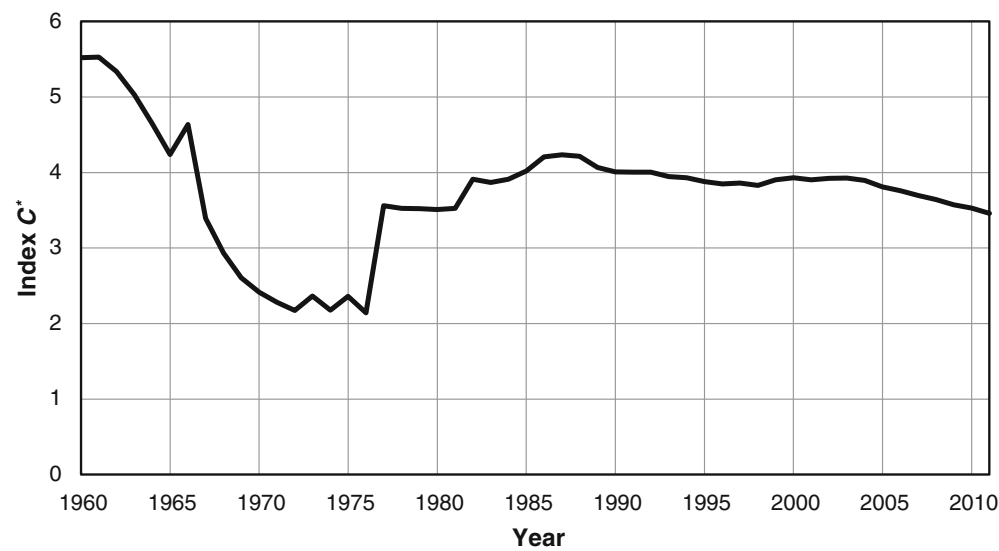

Fig. 3 Index $C^{*}$ of life expectancy

lower than that of both Indonesia and India (see Table 3). In that year, China occupied rank 20, Indonesia rank 21 and India rank 22 in our sample. In 1977, however, both Indonesia and China passed India in terms of GDP per capita, with China moving to rank 21, Indonesia to rank 22 and India dropping to rank 20. Life expectancy increased in all three countries; in China it increased by 0.27 years, and in Indonesia and India by 0.59 years. The population weights of China, Indonesia and India remained virtually unchanged in the two years, with the weight of China decreasing a little, and that of India increasing by about as much.

Let us estimate what would have been the effect of India and Indonesia changing places, and then of India and China changing places, using Eq. 24. We take the average population weights of both years, i.e. $s_{\text {China }} \approx 26.22 \%, s_{\text {Indonesia }} \approx 3.98 \%$ and $s_{\text {India }} \approx 18.35 \%$. For the differences in life expectancy we assume $h_{\text {Indonesia }}-h_{\text {India }} \approx 6.05$ years and $h_{\text {China }}-h_{\text {India }} \approx 14.14$ years. The effect on the index of the India-Indonesia switch can be estimated to be equal to 0.0884 . The effect of China and India switching places in the income distribution, by contrast, is approximately equal to 1.3607 . This is nearly equal to the whole observed change of the index between 1976 and 1977, since $C_{1977}^{*}-C_{1976}^{*}=$

Table 3 China, India and Indonesia in 1976 and 1977

\begin{tabular}{lll}
\hline & 1976 & 1977 \\
\hline & GDP per capita (2005 US \$) / rank & \\
China & $1,227 / 20$ & $1,248 / 21$ \\
Indonesia & $1,258 / 21$ & $1,368 / 22$ \\
India & $1,270 / 22$ & $1,242 / 20$ \\
& Life expectancy (years) & \\
China & 65.99 & 66.26 \\
Indonesia & 57.74 & 58.23 \\
India & 51.69 & 52.28 \\
& Population share (\%) & \\
China & 26.27 & 26.18 \\
Indonesia & 3.97 & 3.99 \\
India & 18.30 & 18.40 \\
\hline
\end{tabular}


$3.5595-2.1407=1.4188$. In other terms, the effect of the China-India switch between 1976 and 1977 is so big that it dwarfs the effects of all other changes combined.

\section{The social gradient among low-income and high-income countries}

Before we discuss these findings a bit deeper, let us see whether we can detect any trends in the socioeconomic inequality of health within the group of low-income countries and within that of high-income countries. When looking at the results, we have to keep in mind that the composition of the two groups may change from year to year. Some countries shift from one group to the other depending on whether their GDP per capita falls below or above the average GDP for the world as a whole. The number of changes is limited, though, and one can say that the two groups have a fairly constant composition. Table 4 provides some descriptive statistics on the two groups at the beginning and the end of the period under consideration.

The evolution of the absolute value of the Concentration index for both groups is represented on Fig. 4. Quite surprisingly, we do not find that the social gradient is always steeper for low-income countries than for high-income countries. Only from 1980 onwards do we find that $C^{*}$ for the group of low-income countries is higher than $C^{*}$ for the group of highincome countries. Between 1960 and 1980 the index is often much lower for the former than for the latter, and in the period 1967-1976 it is even negative, indicating that life expectancy was negatively correlated with GDP per capita in the group of low-income countries. This is the opposite of what the received interpretation of the Preston curve suggests.

The main reason for the low values of the index for the group of low-income countries in the period 1960-1980 is, once again, to be found in the relative positions of China and India. Both are in the group of countries with below average incomes, and they dominate the group even more than they do the world as a whole. For much of the period China had a lower per capita income than India, but a higher life expectancy. Taking into account just these two countries, the gradient would have been negative - at least until 1977, when China surpassed India in terms of per capita income. That these flat or even negative gradients for low-income countries seem to have escaped the attention of most commentators, indicates either that the case of China (relatively low income, relatively high life expectancy) has been handled as an 'exception', or that all countries have been treated alike. Given that after 1980 we do find steeper gradients in low-income countries than in high-income countries may be seen as a justification of that approach, although it remains strange that the largest

Table 4 Summary data on the low-income and high-income groups

\begin{tabular}{lllll}
\hline & 1960 & & 2011 & \\
\hline & Low income & High income & Low income & High income \\
Countries (number) & 68 & 35 & 60 & 43 \\
Population (persons) & $1,773,754,624$ & $740,381,696$ & $4,565,398,016$ & $1,369,830,784$ \\
Population share (\%) & 70.55 & 29.45 & 76.92 & 23.08 \\
GDP/capita (2005 US \$)* & 1,167 & 9,317 & 5,536 & 29,528 \\
Life expectancy at birth (years) & 44.7 & 67.2 & 68.7 & 79.1 \\
\hline
\end{tabular}

Source: World Bank, World Development Indicators and own calculations based on Penn World Table 8.1

* Calculated based on Penn World Table expenditure-side real GDP at chained PPPs 


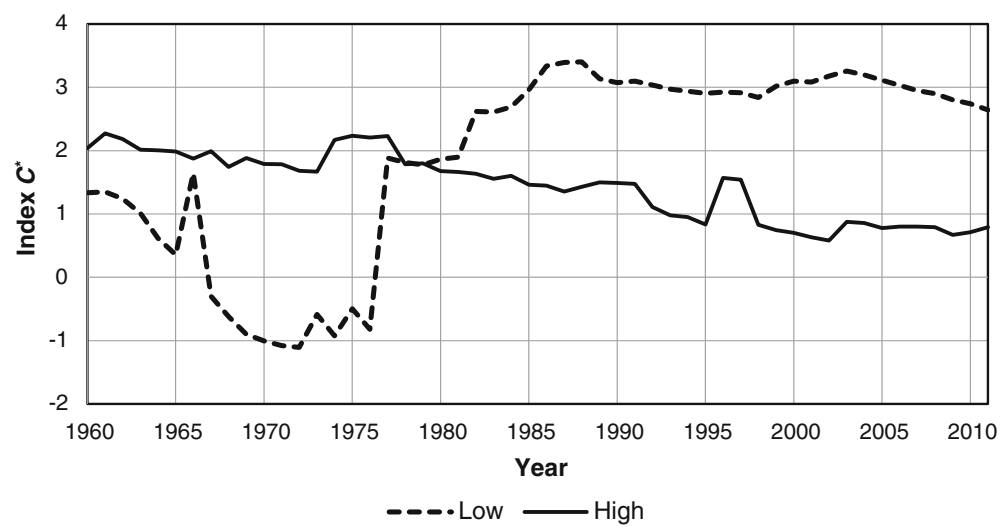

Fig. 4 Index $C^{*}$ for low- and high-income groups

country of the world is either considered as an outlier, or given the same weight as very small countries.

\section{Discussion}

We clearly have a set of puzzling results which call for explanation. Did the global social gradient really change spectacularly in 1977 ? Can it be true that for a considerable period of time the social gradient was lower among low-income countries than among high-income countries? The least we should do is to examine the possibility that these surprising findings are due to a defect in the measuring rod we are using.

Let us begin by the first question. Inequality indices are meant to convey information about the extent of inequality and about changes in inequality. Large increases or decreases should indicate major shifts in the distribution, or in the case of socioeconomic inequality, major shifts in the correlation between income and health. It may be doubted whether such a major shift occurred between 1976 and 1977. The changes in GDP per capita and life expectancy of China and India were relatively small, and yet they produced a massive increase of the index.

It could be argued that the effect was caused by what is allegedly an exceptional constellation of the stars. The shift involved the two largest countries in the world, which is unusual, and it took place when their differences in life expectancy were quite substantial, which is also atypical, since countries with similar income ranks can be expected to have similar life expectancies. This means that everything was in place to make the effect described by Eq. 24 exceptionally large. Surely, if a major shift in inequality occurred between 1976 and 1977 , other indices must reveal the same. To check this, we calculated the values of an alternative index of socioeconomic inequality of health. We chose the $\kappa$ index proposed by Abul Naga and Geoffard (2006: 365). Taking into account the sample weights, the index can be written as follows:

$$
\kappa=\frac{\sum_{i} s_{i} y_{i}^{\alpha} h_{i}^{\beta}}{\sum_{i} s_{i} y_{i}^{\alpha} \sum_{i} s_{i} h_{i}^{\beta}}
$$

where $\alpha$ and $\beta$ are parameters. In their social welfare framework, inspired by the work of Tsui $(1995,1999)$, the values of $\alpha$ and $\beta$ are taken from the social welfare function. If these 


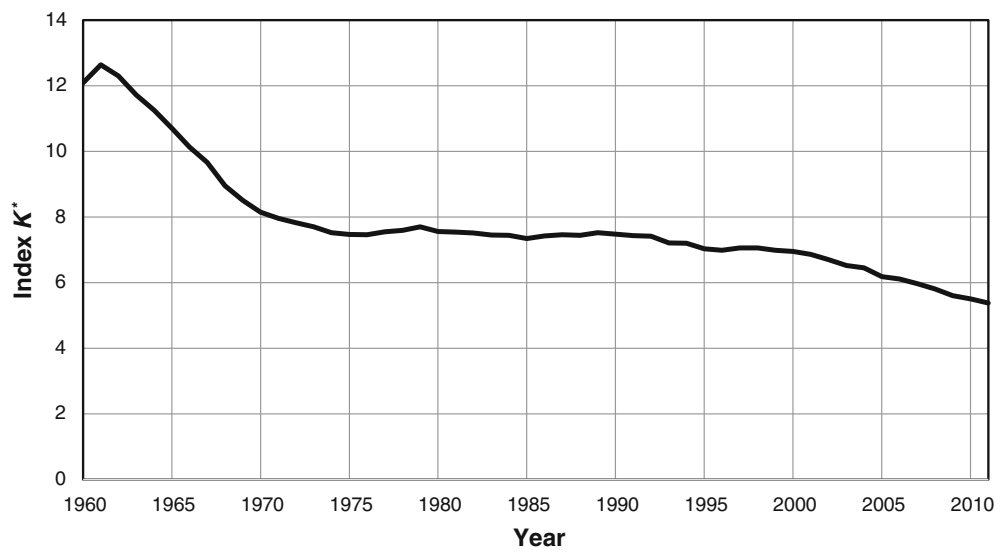

Fig. 5 Index $K^{*}$ of life expectancy

are positive, we must have $\alpha+\beta \leq 1$. For our calculations, however, we have selected the simple parameter values $\alpha=1$ and $\beta=1$, which are outside the range specified by Abul Naga and Geoffard (2006). Although this choice may make it problematic to interpret our results in terms of social welfare, it does not imply that the index is no longer a good measure of association between income and health. ${ }^{6}$

Zhong (2009) has used the $\kappa$ index as an alternative to the Concentration index for the measurement of socioeconomic inequality of health in China. Since the index is greater than 1 in the case of a positive association and smaller than 1 in the case of a negative association (Abul Naga and Geoffard, 2006: 366), Zhong (2009: 1065) suggested we might look at $\ln \kappa$ rather than $\kappa$. We opt for another modification: we look at $K=\kappa-1$ rather than $\kappa$. This is a relative index; its absolute version is $K^{*}=K \mu_{h}$. It is this index $K^{*}$ which we are going to use as an alternative to the absolute Concentration index. Clearly, this alternative index is based on the levels of income, not the ranks. ${ }^{7}$

The results can be found in Fig. 5. The difference with Fig. 3 is striking: the alternative index does not show any sign of the big 1977 bump. Instead, what we see is an almost continuously decreasing trend. In contrast to what $C^{*}$ suggests, the global social gradient of life expectancy does not seem to have increased since 1977, let alone spectacularly so.

As far as the second question is concerned, we can likewise doubt whether the Concentration index really captures the trends within the low-income and high-income groups. Especially the evolution of the index for the low-income group, which includes the two giants China and India, must be treated with a lot of caution. In this case too, we checked what the alternative index revealed about the trends (Fig. 6). For the high-income group the two indices give more or less the same result: a slowly decreasing trend, with ups and downs along the road. For the low-income group, we do not find negative values when using index

\footnotetext{
${ }^{6} \mathrm{We}$ checked the robustness of our results using the parameter values $\alpha=\beta=0.5$. No major changes in trends were detected. The results of the calculations can be obtained upon request from the authors.

${ }^{7}$ Index $K^{*}$ is equal to the absolute version of the level-dependent index recently proposed by Erreygers and Kessels (2015). They show that this index reacts continuously to changes in incomes, while the Concentration index, as noted above, does not.
} 


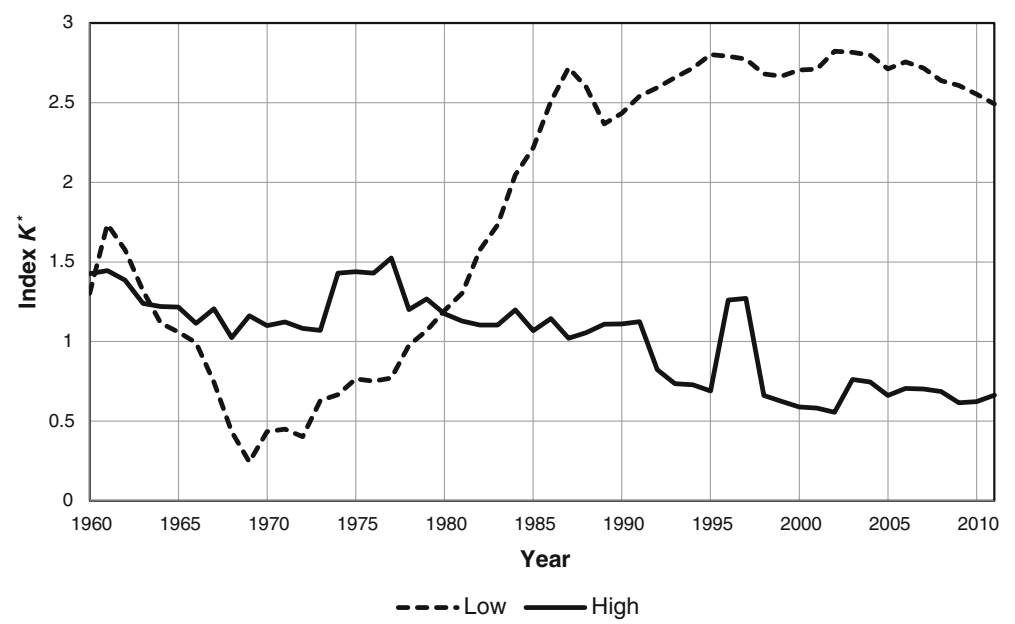

Fig. 6 Index $K^{*}$ for low- and high-income groups

$K^{*}$. But for the rest the broader trends are similar: decreasing and mostly relatively low levels of inequality in the 1960s and 1970s, and relatively high levels of inequality from the 1980s onwards.

Another way of comparing the two indices consists of decomposing the indices into a between- and a within-component. The between-component measures the extent of inequality between the low- and the high-income group, assuming that all countries in each group have the same GDP per capita and the same life expectancy, equal to their group average. The within-component is a weighted average of the inequality within the two subgroups. In the case we are considering here, both indices can be decomposed

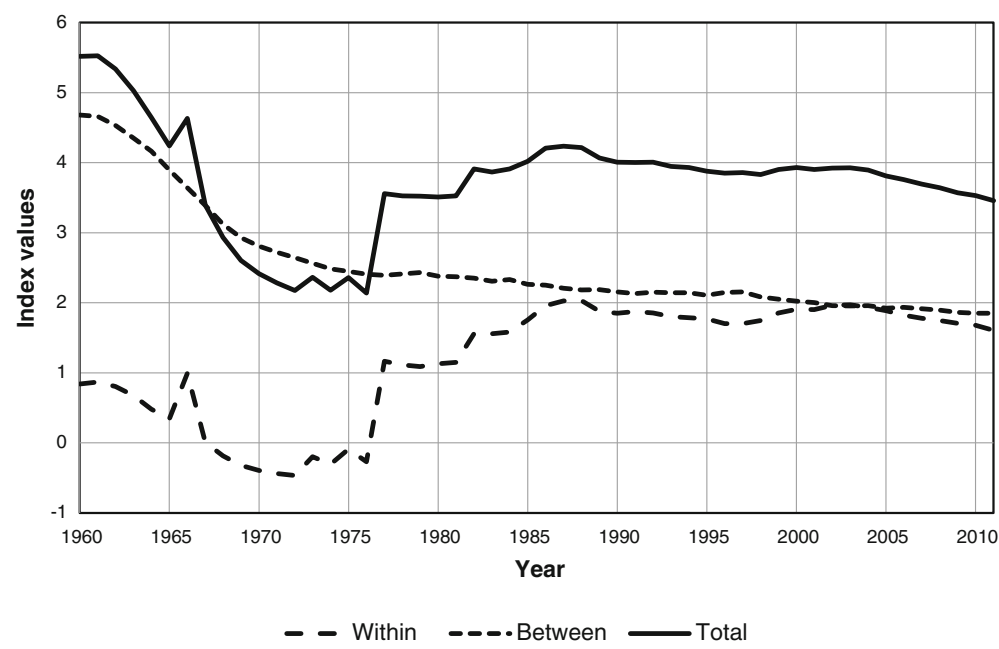

Fig. 7 Decomposition of index $C^{*}$ 


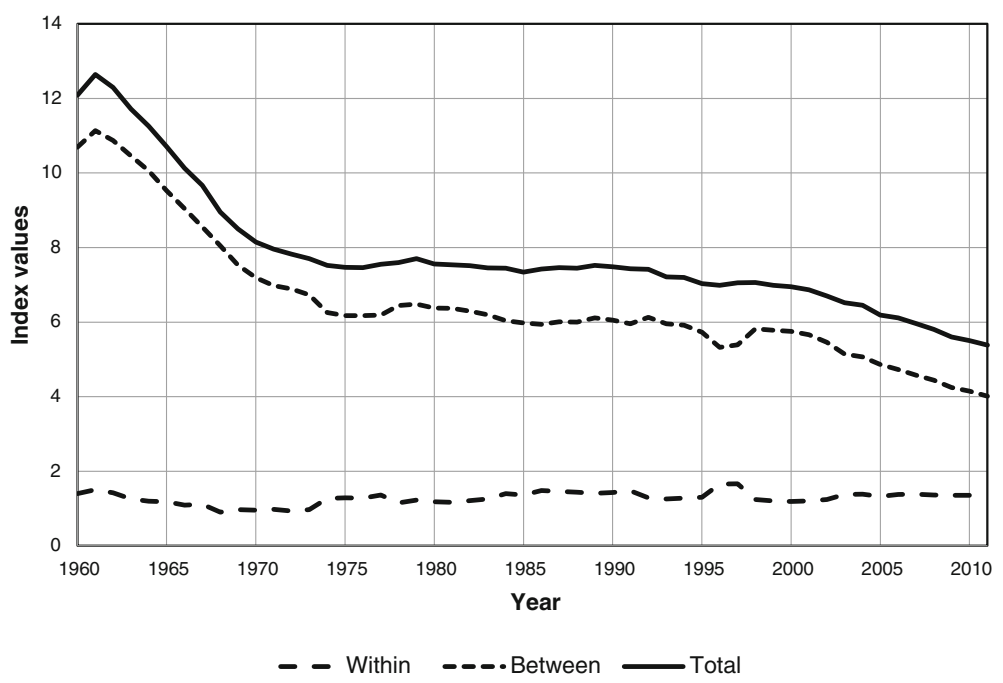

Fig. 8 Decomposition of index $K^{*}$

in this way without a residual term. ${ }^{8}$ Figures 7 and 8 represent the decomposition results for the two indices. According to the absolute version of the Concentration index, the between-component decreased almost constantly over the years, while the withincomponent fluctuated very much. From the end of the 1980s onwards, the contributions of the between- and within-components are nearly identical. The downward trend of the between-component is also detected by the alternative index $K^{*}$. According to this index, however, the within-component is almost stable over time, and moreover is much smaller than the between-component.

It seems useful to observe that the issue we are dealing with here is specific to so-called 'bivariate' rank-dependent indicators. ' 'Univariate' rank-dependent inequality indicators, such as the Gini coefficient, do not suffer from this problem since they take into account both the ranks and the levels of the variable under consideration. As an illustration, let us look at the evolution of the cross-country Gini coefficient of income inequality for the same period and the same group of countries we considered earlier (Fig. 9). The Gini coefficient has remained remarkably stable over time and does not show any striking or suspicious change in 1977. The change in income ranks between China and India produces hardly a ripple on the surface.

Finally, we point out that the issue is related to, but distinct from, the grouped data problem studied by Clarke and Van Ourti (2010). They focus on the bias caused by estimating

\footnotetext{
${ }^{8}$ It should be noted, however, that the weights to be used for the calculation of the within-component are not the same in both cases. For the absolute version of the Concentration index, the respective weights for the levels of inequality in the low- and high income groups are $\left(s_{L}\right)^{2}$ and $\left(s_{H}\right)^{2}$, where $s_{L}$ is the population share of the low-income countries and $s_{H}$ that of the high-income countries. For index $K^{*}$ the weights are $s_{L}\left(\mu_{y, L} / \mu_{y}\right)$ and $s_{H}\left(\mu_{y, H} / \mu_{y}\right)$, where $\mu_{y, L}$ stands for the average GDP per capita in the low-income group and $\mu_{y, H}$ for that in the high-income group. Observe that $s_{L}\left(\mu_{y, L} / \mu_{y}\right)+s_{H}\left(\mu_{y, H} / \mu_{y}\right)=1$, while $\left(s_{L}\right)^{2}+\left(s_{H}\right)^{2}<1$.

${ }^{9}$ Although the term 'bivariate inequality' may be interpreted in different ways, it is increasingly used in the context of socioeconomic inequality of health; see, e.g., Brekke and Kverndokk (2002).
} 


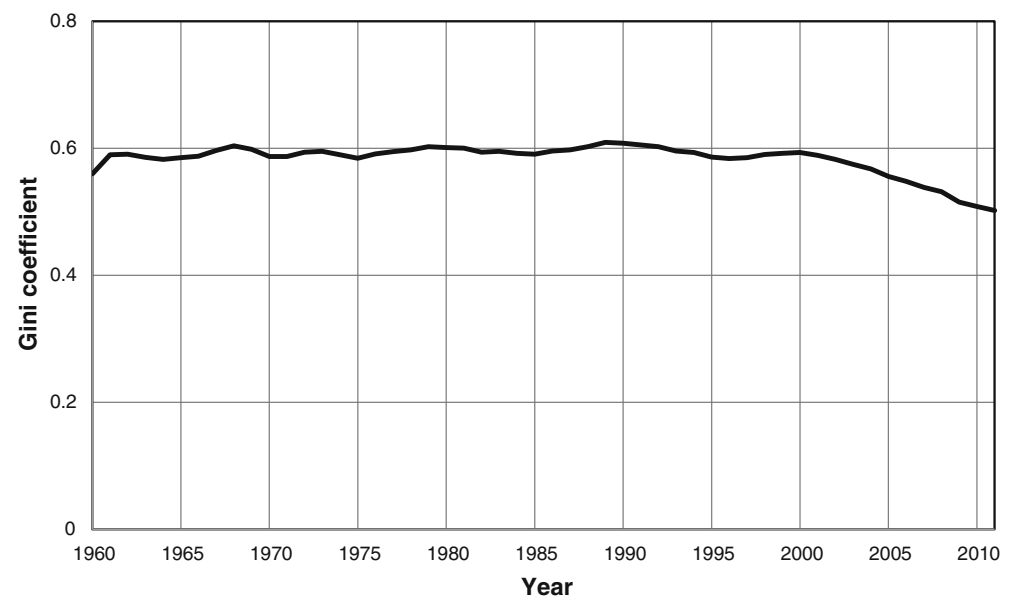

Fig. 9 Gini coefficient

the extent of inequality on the basis of aggregated data (e.g., relating to income groups) rather than on the basis of micro-data. Our concern here is with the correct estimation of the between-group inequality only; in the absence of reliable individual data on income and health, we cannot know to what extent this inequality is a good approximation of the 'true' amount of inequality. ${ }^{10}$

\section{Detecting the problem}

Our empirical study and the comparison of the two indices reveal the extreme sensitivity of rank-dependent indicators of socioeconomic inequality of health to small changes involving a rank switch between countries with large population weights. In cross-country applications, indices such as the Concentration index may show signs of trend reversals or of major shifts which are not detected by other indices. ${ }^{11}$ It is the combination of large population weights and rank-dependency which can produce spurious results.

The issue identified here appears to be relevant for any empirical study that uses weights. Differences in weights are most likely to show up in the context of international and regional studies, since there is substantial variety in the sizes of countries and regions. The problem may also affect studies based on individual data, e.g. surveys using sampling weights, provided some of the weights are substantially larger than others. As we have shown in our study of inequalities in life expectancy across countries, relatively few observations can represent a significant proportion of the overall sample. Although they have a potentially great effect on the measured degree of inequality, as long as their weights reflect real differences and not measurement errors, they should not be treated as 'outliers'.

It would be useful to have a diagnostic test to see how sensitive the Concentration index is to small changes in incomes of different observations in the sample under analysis. The

\footnotetext{
${ }^{10} \mathrm{As}$ far as we know, the only study which would provide a good basis for such a comparison is the one by Wu, Stavvides and Stengos (2014).

${ }^{11}$ Using the relative versions of the indices does not change the conclusions.
} 
aim of such a test is not to predict whether and when a major shift in the index is bound to occur. What it can tell us, is whether changes in the situation of particular countries could have a major impact on the index.

One intuitive measure of sensitivity would be to estimate the elasticity of the Concentration index to changes in income of each observation in the sample. Since a change in income can generate a variety of changes in ranks (from no change at all to changes involving several countries), the elasticity has to be estimated empirically. A straightforward algorithm for calculating this elasticity involves setting a proportional change parameter $\rho$ which is then applied sequentially across the observations. Since we are interested in the effect of both positive and negative changes of income, for each observation we need to look at the effect of decreasing $y_{i}$ to $y_{i}^{l}=(1-\rho) y_{i}$ as well as at that of increasing $y_{i}$ to $y_{i}^{u}=(1+\rho) y_{i}$. Let us denote the new values of the index respectively by $C_{i}^{l *}$ and $C_{i}^{u *}$, and the changes by $\Delta C_{i}^{l *}=C_{i}^{l *}-C^{*}$ and $\Delta C_{i}^{u *}=C_{i}^{u *}-C^{*}$. This gives us two elasticity estimates, $\varepsilon_{i}^{l}=\frac{\Delta C_{i}^{l *}}{-\rho y_{i}} \cdot \frac{y_{i}}{C^{*}}=\frac{C^{*}-C_{i}^{l *}}{\rho C^{*}}$ and $\varepsilon_{i}^{u}=\frac{\Delta C_{i}^{u *}}{\rho y_{i}} \cdot \frac{y_{i}}{C^{*}}=\frac{C_{i}^{u *}-C^{*}}{\rho C^{*}}$. We could, however, also look at the combined effect of the two changes on the index, i.e. at the effect of a hypothetical change from $y_{i}^{l}$ to $y_{i}^{u}$. In that case, the change in income is equal to $2 \rho y_{i}$ and the change of the index equal to $\Delta C_{i}^{*}=C_{i}^{u *}-C_{i}^{l *}$. This gives a third estimate of the elasticity: $\varepsilon_{i}=\frac{\left(C_{i}^{u *}-C_{i}^{l *}\right)}{2 \rho y_{i}} \cdot \frac{y_{i}}{C^{*}}=\frac{\left(C_{i}^{u *}-C_{i}^{l *}\right)}{2 \rho C^{*}}$. It is easy to see that this is nothing but the average of the two other elasticities: $\varepsilon_{i}=\left(\varepsilon_{i}^{l}+\varepsilon_{i}^{u}\right) / 2$.

As an illustration, we calculated the elasticities $\varepsilon_{i}$ for all 103 countries of our sample for the year 1976, i.e. the year just before the dramatic surge in the cross-country Concentration index. We chose a value of $\rho$ equal to $4 \%$; this value is slightly above the weighted average growth rate of per capita income for the countries in our sample between 1975 and 1976 (3.27\%), and seems indicative of changes which might occur in a country's per capita GDP in a given year. The results are represented in Fig. 10. What is immediately obvious is that two countries have exceptionally high elasticities: China and India. The large positive elasticity for China is due to the effect which an increase of its per capita income would

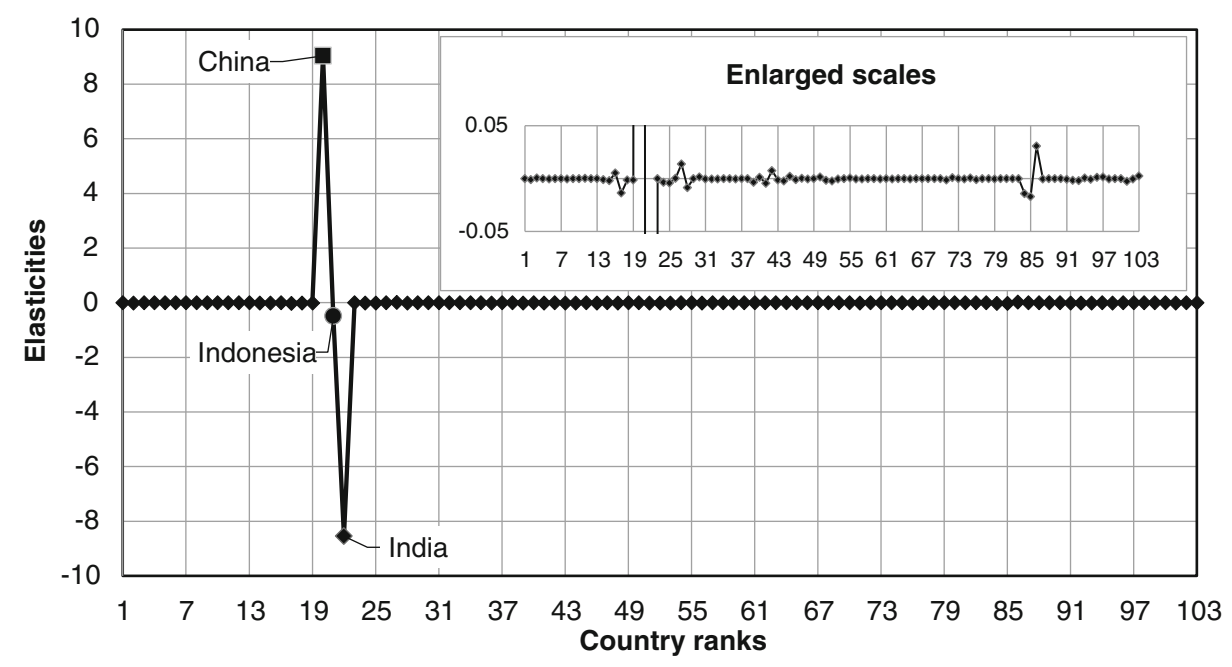

Fig. 10 Elasticities for 1976 
have; in other words, China has a very high and positive value of $\varepsilon_{C h i n a}^{u}$. The large negative elasticity of India can be explained by the effect which a decrease of its per capita income would have; i.e., India has a very high and negative value of $\varepsilon_{\text {India }}^{l}$. The two are, of course, strictly related: in both cases, the large impact on the Concentration index comes from the fact that these income changes would entail a rank change between China and India.

\section{Conclusion}

This note shows that rank-dependent indices of socioeconomic inequality of health may behave oddly when they are used to measure the evolution of cross-country inequality over time. When rank-dependent indices such as the Concentration index are applied to samples in which some observations have very large sample weights, they are liable to overreact to small changes in the socioeconomic distribution which involve the largest countries, i.e. the observations with the largest sample weights. The main reason is not so much that the indices depend upon the ranks of the socioeconomic variable, but rather that they do not take into account the levels of this variable. This is illustrated by the fact that the Gini coefficient of income inequality, which is a measure based upon both the ranks and the levels of income, does not have the hypersensitivity of the Concentration index. Moreover, alternative indices of socioeconomic inequality of health which do take the levels of the socioeconomic variable into account, do not suffer from the same weakness. When it comes to measuring socioeconomic inequality of health between countries, therefore, using a level-dependent index seems to be a safer choice than using a rank-dependent index. This concerns mainly future research, since up to now the Concentration index has been applied to only a handful of cases of between-country inequality. The main difficulty is the scarcity of data, as pointed out by $\mathrm{Wu}$, Stavvides and Stengos (2014: 257). When more data on income and health become available, it is to be expected that more attempts will be made to estimate indices of socioeconomic inequality.

The simple diagnostic test we propose in the paper can be used to determine empirically whether a sample may give rise to large swings in the Concentration index as a result of small changes in income levels. It cannot determine whether a swing will actually occur; that depends upon the specific nature of the changes. If the test reveals that the Concentration index is highly sensitive to changes in income of one or more countries (or, more generally, of one or more observations), it seems better to avoid using the Concentration index.

Acknowledgments We thank Paul Makdissi, Tom Van Ourti and Michael Zyphur for their observations on a previous version of the paper. We are also grateful to seminar audiences at the School of Population and Global Health of the University of Melbourne and at the Centre for Social Policy of the University of Antwerp, and to participants of the North American Productivity Workshop 2016 in Québec. The detailed comments of two anonymous reviewers and of the editor of the journal have been very helpful. All errors are our responsibility.

Open Access This article is distributed under the terms of the Creative Commons Attribution 4.0 International License (http://creativecommons.org/licenses/by/4.0/), which permits unrestricted use, distribution, and reproduction in any medium, provided you give appropriate credit to the original author(s) and the source, provide a link to the Creative Commons license, and indicate if changes were made. 


\section{References}

Abul Naga, R.H., Geoffard, P.-Y.: Decomposition of bivariate inequality indices by attributes. Econ. Lett. 90, 362-367 (2006)

Brekke, K.A., Kverndokk, S.: Inadequate bivariate measures of health inequality: The impact of income distribution. Scand. J. Econ. 114, 323-333 (2002)

Clarke, P., Van Ourti, T.: Calculating the concentration index when income is grouped. J. Health Econ. 29, 151-157 (2010)

Creedy, J.: A note on computing the Gini inequality measure with weighted data. Working Papers in Public Finance, Working Paper 03/2015, Victoria Business School. Victoria University of Wellington, New Zealand (2015)

Deaton, A.: The Great Escape: Health, Wealth, and the Origins of Inequality. Princeton Princeton University Press (2013)

Decancq, K., Decoster, A., Schokkaert, E.: The evolution of world inequality in well-being. World Devel 37, 11-25 (2009)

Dorling, D., Shaw, M., Davey Smith, G.: Global inequality of life expectancy due to AIDS. Brit. Med. J. 332, 662-664 (2006)

Erreygers, G., Kessels, R.: Socioeconomic status and health: A new approach to the measurement of bivariate inequality. Antwerp, University of Antwerp, Faculty of Applied Economics, Working Paper 2015/017 (2015)

Erreygers, G., Van Ourti, T.: Measuring socioeconomic inequality in health, health care and health financing by means of rank-dependent indices: A recipe for good practice. J. Health Econ. 30, 685-694 (2011)

Hajizadeh, M., Sia, D., Heymann, S.J., Nandi, A.: Socioeconomic inequalities in HIV/AIDS prevalence in sub-Saharan African countries: Evidence from the Demographic Health Surveys. Int. J. Equity Health 13, 18 (2014)

Hoffmann, R.: Effect of the rise of a person's income on inequality. Braz. Rev. Econom. 21, 237-262 (2001)

Lambert, P.J., Lanza, G.: The effect on inequality of changing one or two incomes. J. Econ. Inequal. 4, 253-277 (2006)

Lerman, R.I., Yitzhaki, S.: Improving the accuracy of estimates of Gini coefficients. J. Econom. 42, 43-47 (1989)

Milanovic, B.: Global income inequality in numbers: In history and now. Glob. Policy 4, 198-208 (2013)

O’Donnell, O., Van Doorslaer, E., Wagstaff, A., Lindelow, M.: Analyzing Health Equity Using Household Survey Data. The World Bank, Washington DC (2008)

Preston, S.H.: The changing relation between mortality and level of economic development. Popul. Stud. 29, 231-248 (1975)

Reidpath, D.D., Allotey, P.: Measuring global health inequity. Int. J. Equity Health 6, 16 (2007)

Sala-i-Martin, X.: The world distribution of income: Falling poverty and convergence, period. Quart. J. Econ. 121, 351-397 (2006)

Tsui, K.-Y.: Multidimensional generalizations of the relative and absolute inequality indices: The AtkinsonKolm-Sen approach. J. Econ. Theory 67, 251-265 (1995)

Tsui, K.-Y.: Multidimensional inequality and multidimensional entropy measures: An axiomatic derivation. Soc. Choice Welfare 16, 145-157 (1999)

Wagstaff, A., Paci, P., Van Doorslaer, E.: On the measurement of inequalities in health. Soc. Sci. Med. 33, 545-557 (1991)

Wu, X., Savvides, A., Stengos, T.: The global joint distribution of income and health. In: Ma, J., Wohar, M. (eds.) Recent Advances in Estimating Nonlinear Models. With Applications in Economics and Finance. Springer, New York (2014)

Zhong, H.: A multivariate analysis of the distribution of individual's welfare in China: What is the role of health? J. Health Econ. 28, 1062-1070 (2009) 